\title{
CHOOSING FILTERS TO MAKE CCD PHOTOMETRY TRANSFORMABLE TO OTHER DETECTORS
}

\author{
Andrew T. Young \\ San Diego State University
}

\begin{abstract}
Transforming CCD data is difficult. Until recently, the general transformation problem was so poorly understood that there were no established design criteria. Now, the Hilbert-space approach to photometry tells us to match the standard passbands in the leastsquares sense.
\end{abstract}

Unfortunately, the spectral responsivities of CCDs vary much more than do conventional detectors, and must be known to design filters. Each individual chip generally requires its own set of filters, if results are to be better than second-rate. Even so, spatial variations in spectral response limit the accuracy that can be reached, unless color terms are determined for individual pixels. Such CCD calibration problems involve the generally neglected optical properties of CCDs and the systems in which they are used.

Large observatories will have to establish calibration laboratories, and to abandon the common practice of moving "standard" filter sets from one instrument to another. Smaller institutions will need to purchase the services of such calibration laboratories if they are to produce accurate CCD photometry. These are major changes in the way astronomers are accustomed to working; but such changes are necessary to stem the backlash developing against CCD photometry.

\section{INTRODUCTION}

While CCD photometry can reach a precision better than a millimagnitude, provided that Herculean efforts are made to keep star images fixed on the chip, and to cancel a host of systematic errors (Gilliland et al. 1993), the accuracy reached is usually inferior to that of ordinary photoelectric photometry. For example, although Gilliland et al. (1991) previously achieved millimagnitude precision, the accuracy reached was an unspectacular 0.018 to 0.038 mag per standard star. In their latest effort, Gilliland et al. (1993) did not even attempt to produce standardized photometry on any existing system, but deliberately sacrificed accuracy to obtain the highest possible precision.

However, many problems require accuracy as well as (or even instead of) precision. Any application in which color indices are used to classify stars requires accurate colors. Distance determination is a prime example: corrections must be made for reddening, so the observations must be referred to a standard system.

Transforming CCD instrumental magnitudes to standard systems is difficult (Bessell 1990, 
1993a, b). Indeed, until recently, the general understanding of transformations was so poor that there was neither an established criterion for reproducing a photometric system with a new detector, nor a sound theoretical basis for transforming data taken with any instrument to a standard system. Transformations have been done entirely empirically, using one or more convenient color indices (and perhaps other parameters, such as estimates of reddening or metallicity) as independent variables, without regard for any physical model.

As long as they used instrumentation similar to that which defined a system, most observers managed to ignore the complexities of transformations. However, now that redsensitive detectors are commonplace, transformation errors are larger, and the inadequacy of a purely empirical approach is more evident. Transformation errors due to mismatched passbands - the so-called conformity errors (Manfroid and Sterken 1992) - often exceed a tenth of a magnitude, and can no longer be ignored.

\section{TRANSFORMATIONS}

The standard mythology holds that transformations "ought" to be linear and single-valued, although King (1952) showed more than 40 years ago that this cannot be true; the only linear, single-valued transformation is the identity transformation, when the instrumental passbands exactly match the standard ones. Nevertheless, wishful thinking continues to triumph over scientific analysis. Recent attempts to reproduce older photometric systems with new detectors have re-emphasized the importance of nonlinear terms (cf. Bessell 1990), and the multivaluedness of transformations - especially for reddened or chemically peculiar stars - has long been known.

The extension of the standard Strömgren-King theory to higher-order terms (Young 1992a) revealed serious weaknesses in the series expansion on which it is based. First, convergence of the series is exceedingly slow, so that terms depending on third- and fourth-order central moments are easily observable. Second, high-order terms are made worse by the nonlinearity of converting from intensities to magnitudes. Third, this nonlinearity mixes together terms of all orders, so that both nonlinear and multivalued effects are inherent in transformations based on color indices.

The linear color term in transformations is proportional to the difference between the centroid wavelengths of the instrumental and standard bands. But higher-order terms depend on not only the difference of higher central moments, measured from a common reference wavelength, but also powers and cross-products of low-order spectral derivatives (Young, 1992a). Consequently, while a small linear color term is usually necessary to obtain small higher-order terms, it is by no means sufficient. The rule that color coefficients must be kept below 0.1 is a useful guide, but is not adequate to guarantee accurate transformations.

To indicate the size of higher-order terms, consider the two passbands shown below (Fig. 1). The passband in the upper half of the figure consists of two rectangular portions (shaded), and the lower, of three. Together, the two passbands add up to a single rectangular function, indicated by the dashed outline.

Both passbands are symmetric about the same wavelength, so they have the same centroid, and all their odd-order central moments vanish. Also, they have been constructed so that their 


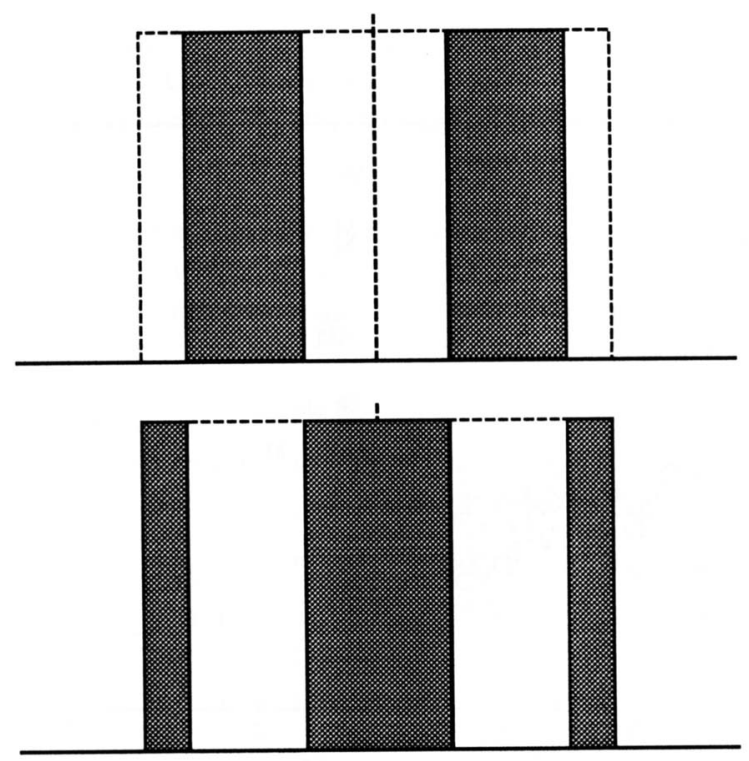

Fig. 1. Two passbands (shaded) having the same centroid and second moment (effective band width). Note that the two passbands do not overlap, and so are orthogonal.

second moments are equal. This means that the "band width" that appears in the classical series expansion (King 1952, Young 1992a) is the same for both bands. Furthermore, their fourth moments differ by less than $20 \%$; so any differences between measurements made with these bands must be mainly due to $6^{\text {th- }}$ and $8^{\text {th }}$-order terms. Given these similarities, one might not expect that measurements made with the two passbands could differ very much. To see how large such differences can be, I have scaled the passbands of Fig. 1 to a width of $124 \mathrm{~nm}$ and a center wavelength of $441 \mathrm{~nm}$, so that each is an approximation to the B band, having the same centroid and mean-square width as $\mathrm{B}$. The bands were then multiplied by each of the stellar spectral irradiance functions tabulated by Gunn and Stryker (1983) and integrated, and the magnitude differences between the two simulated passbands were calculated and plotted as a function of $(\mathrm{B}-\mathrm{V})$.

Each band averages over $62 \mathrm{~nm}$ of spectrum, so one might expect stellar spectral features to be largely averaged out. If high-order terms were negligible, the two magnitudes should be very similar, and the plot of magnitude difference would be a horizontal straight line.

Nevertheless, as Fig. 2 shows, the differences span a tenth of a magnitude for $\mathrm{K}$ stars, and more than $0.2 \mathrm{mag}$ for late $\mathrm{M}$ stars. Even for earlier spectral types, there are large nonlinearities, and significant spread (multivaluedness due to conformity error). Among the A stars, we see effects due to luminosity and metallicity that amount to $0.06 \mathrm{mag}$. If one of these passbands were a standard band, and the other were the corresponding instrumental one, there would be serious difficulties in transforming between them, in spite of their equal effective wavelengths and band widths. These large differences are Manfroid and Sterken's "conformity errors". Evidently, stellar spectra are complicated enough that the terms due to their high-order derivatives are quite substantial. 


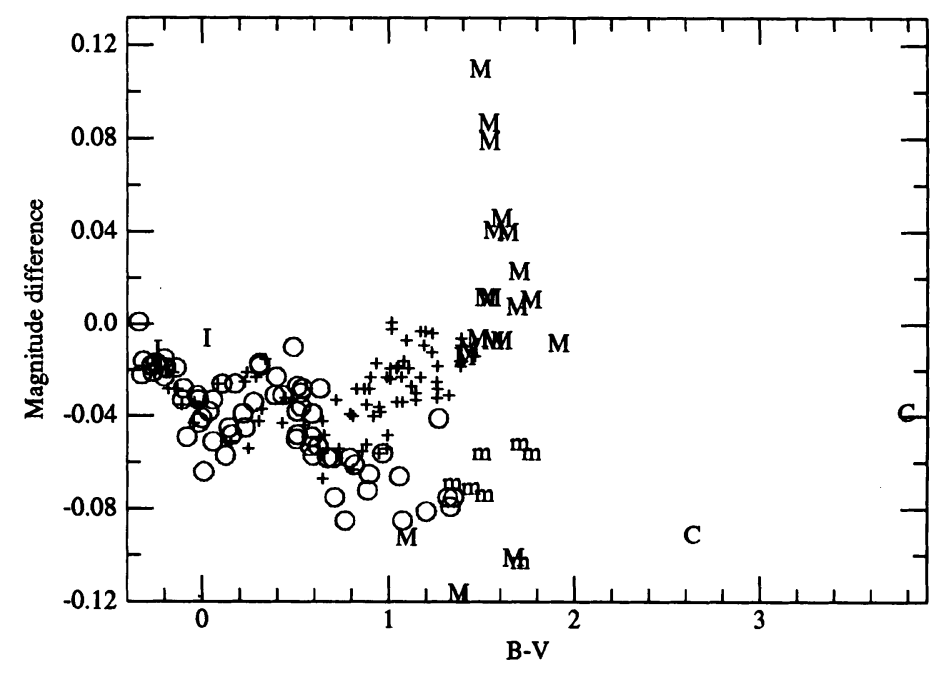

Fig. 2. Difference between magnitudes measured through the two passbands of Fig. 1, scaled to match the centroid and second moment of the B band.

Furthermore, the higher-order derivatives of the (smoothed) stellar spectral energy distribution that appear in the theory are badly estimated by conventional color and curvature indices, which suffer from severe aliasing due to undersampling of the spectrum. As I emphasized at the previous General Assembly (Young 1992b), this means that the information required to do accurate transformations is never acquired in the first place in conventional photometry.

In an effort to avoid the nonlinearities introduced by using magnitudes and colors, I have recently adopted a more fundamental point of view (Young 1994). What we are trying to measure is the spectral energy distribution of a star's light; this is a function, not a scalar quantity. What we actually measure is a function of this function, namely the functional

$$
L=\int_{0}^{\infty} I(\lambda) R(\lambda) d \lambda
$$

which is a scalar quantity that depends on $R(\lambda)$, our instrumental function. At best, we can measure a few such scalar values through a small set of passbands.

If we regard the functions $I(\lambda)$ and $R(\lambda)$ as vectors in Hilbert space, the measured "intensity" is the projection of $I$ on R. A set of instrumental passbands can be regarded as basis vectors that define a space of low dimensionality. Functional analysis then shows that our 
conformity errors are proportional to the sine of the angle between one of the standard basis vectors and its projection into our instrumental space. This projection is simply the linear combination of our instrumental vectors that approximate the standard vector in the leastsquares sense.

From this point of view, the problem with the passbands shown in Fig. 1 is that they are orthogonal to each other; they measure entirely different spectral regions. Even though these regions are interleaved, they do not overlap. The large conformity errors shown in Fig. 2 are caused by the orthogonality of the two response functions in Fig. 1.

To minimize conformity errors, we must choose a set of instrumental response functions so that a linear combination of them is a good least-squares approximation to each standard response function. As Fig. 2 shows, just matching low-order moments is not enough; the instrumental passbands must accurately match the standard ones in all details. This rule provides a simple and reliable filter-design criterion for reproducing any photometric system with a new detector whose spectral responsivity is known. It also shows that using a detector with unknown spectral response is a recipe for failure.

Furthermore, as the intensity that would have been measured with the standard passband is the same linear combination of the intensities measured with the instrumental passbands, the Hilbert-space view provides a simple, linear transformation algorithm for data reduction. (If we want magnitudes and colors, we can take logarithms of the intensities after transforming them to the standard system.)

Good approximations require instrumental passbands with substantial overlap. However, such overlapping passbands are needed in any case to avoid aliasing problems due to undersampling the stellar spectrum. Future photometric systems will provide proper sampling, but at present one must deal with undersampled systems, where conformity errors are inevitable and uncorrectable. The only way to minimize the damage is to minimize the mean-square difference between the instrumental and the standard passbands. At least, we now know what to strive for.

Numerical experiments show that conformity errors for "ordinary" stars are typically about a tenth of the sine of the angle between instrumental and standard response vectors; the angle is typically 10 degrees or so, for conventional detectors, and the transformation errors are typically 0.01 or 0.02 mag (Beckert and Newberry 1989). Of course, for stars with spectral peculiarities, the errors can be an order of magnitude larger (comparable to the sine of the angle). However, for CCDs, the angles for bands like U and I can be on the order of 15 degrees. Thus we expect conformity errors on the order of 0.03 mag for ordinary stars, and tenths of a magnitude for peculiar stars.

These conformity errors are systematic errors for individual stars that depend on the properties of individual stellar spectra. Although they look like "noise" in plots like Fig. 2, they cannot be reduced by making more measurements, using a larger telescope, or measuring more standard stars. 


\section{MATCHING UNDERSAMPLED SYSTEMS}

Some systems were originally defined by the properties of available detectors such as the eye and the unsensitized photographic plate; later, the red and blue cutoffs of photomultipliers such as the 1P21 were used. The spectral response of silicon detectors is quite different from these, so the difference must be made up by proper filtering. In particular, the large infrared response of silicon photodiodes, combined with the low effectiveness of infrared-absorbing glasses, greatly exacerbates red-leak problems.

CCDs differ from photomultipliers in a second, important way. The spectral response of a photocathode depends on only a few factors: the band gap of the material, the electron affinity, and the thickness of the material are the most important. In contrast, the spectral response of a simple silicon photodiode depends on about a dozen different parameters (Geist et al. 1982, Schaefer 1984); the optical effects of the electrodes used in CCDs (Kesler and Lomheim 1986) introduce many more parameters. If the device is coated or treated to enhance the blue response, still more parameters affect the spectral response.

Consequently, even nominally similar CCD devices differ considerably more in spectral response than do photomultipliers. Furthermore, the variety of construction and use configurations (front or back illumination; thick or thin; surface or buried channel; various blue-enhancement techniques) produces a much wider variety of spectral responses than are available in photocathodes. Therefore, as has been repeatedly emphasized by Bessell (1993a, b), "it is best to use different glass filter recipes for different CCDs." Walker (1993) gives some particular examples.

The wide range in CCD spectral responses means that the problem of transforming CCD photometry cannot be solved by replacing existing photometric systems by a new "CCD system", as has sometimes been suggested. Because the actual spectral response of a CCD can be quite different from another nominally identical device (see, for example, Schumann and Lomheim 1989), the design of satisfactory filters for matching any standard photometric system requires that each chip's response be individually measured. Few observatories are able to make such measurements today.

Furthermore, the factory tolerances on even the best colored glasses are so lax that they correspond to more than a factor of two in thickness for most of the sharp-cutoff glasses, which are the ones that cause the largest conformity errors. A specification such as " 2 mm of GG 495 " may in fact be satisfied by something between 1 and $4 \mathrm{~mm}$ of GG 495, depending on the particular sample. Here again, accurate spectral-transmittance measurements, free of scattered light, must be made on the actual piece of glass to be used, before the correct thickness can be calculated.

\section{SPATIAL VARIATIONS}

An equally serious problem, which seems to have been almost completely neglected, is the marked variation in spectral response among pixels of a single CCD chip. Because the chip is inherently a multilayer optical device, within which standing waves form, the response of a given pixel depends on the strength of the standing wave in its active volume. Because of the high refractive index of silicon, and the high reflectance of any metallic layers in the electrode 
structure, the contrast of the Edser-Butler fringes formed within the chip can be tens of per cent. Anti-reflection coatings can reduce fringe contrast, but are effective only in a restricted wavelength range; because CCDs are used over at least a factor of two in wavelength, fringe problems cannot be completely eliminated.

Furthermore, because the multilayer structure of a CCD is several wavelengths thick, the fringes formed are of moderately high order, on the order of 10 or 20 . That means that small variations in the thickness of any given layer can make large changes in the fringe phase. Kesler and Lomheim (1986) show an example in which a change in thickness of one layer from 300 $\mathrm{nm}$ to $350 \mathrm{~nm}$ suffices to reverse the phase of fringes that have a period of about $200 \mathrm{~nm}$ in the spectrum. Thus, while one pixel in the device could be twice as sensitive at $750 \mathrm{~nm}$ as at 650 , the reverse could be true for another pixel in the same device. In such a CCD, pixel-to-pixel variations of spectral response within the passband of a typical broadband filter are quite serious.

When a CCD is illuminated by monochromatic light, the Edser-Butler fringes are aliased into the spatial domain by spatial variations in the layer thicknesses. This is the origin of the notorious night-sky fringes often observed at long wavelengths. However, observers who treat the fringes as a flatfielding problem fail to recognize that the night-sky fringes are really only an alias of the real problem, which is strong and rapid variations in spectral response across the chip.

Moreover, the observed contrast of the night-sky fringes is only a lower limit to the inherent contrast of the Edser-Butler channels, for two reasons. First, the filter passband usually contains several significant night-sky lines, whose fringes are more or less out of phase with one another. Wavelength averaging can then greatly reduce fringe contrast in wide-band images, though they may still be visible in narrow-band images. For example, Gullixson (1992) shows dome flats that are fringeless in $\mathrm{R}$ and $\mathrm{I}$, but show pronounced fringing at $\mathrm{H}$-alpha.

Second, because the fringes are of moderately high order, they are smoothed out to some extent by the range of angles subtended by the pupil at the detector. While the pupil is completely filled by an extended source like the night sky or a flatfield exposure, it is not filled by a star image that is not perfectly focused. Each pixel in the chip can be regarded as a twodimensional Foucault tester; if the image is slightly out of focus, only a portion of the pupil will be seen by a particular pixel. Defocusing therefore reduces the solid-angle averaging of the Edser-Butler fringes. Because a star image does not illuminate the detector in the same way as a flatfield, the basic principle that calibration and measurement exposures be made in the same way is violated, and trouble may be expected. Perhaps some flatfielding problems come from variations in focus across the chip.

Third, because stars are generally unresolved, starlight is spatially coherent across the entire telescope pupil, while extended sources like the night sky and calibration targets are incoherent. Thus, the electrode structure of a CCD chip can act as a lamellar grating and produce fringes in starlight that have much less contrast for extended sources. Once again, the flatfielding sources are not comparable to stars, and can fail to show fringes that are significant for star images.

If a CCD shows night-sky fringes in some passband, it is prudent to assume that pixel-to- 
pixel spectral-response variations are at least as large for stars as the sky-fringe contrast. Then, to obtain accurate transformations, one should determine at least a color term for each pixel individually; an average for the entire chip is not likely to be adequate. It might be possible to determine such color terms by observing both "red" and "blue" flatfields for each passband affected by internal fringes. This problem is briefly alluded to by Massey and Jacoby (1992) in connection with large-scale flatfielding differences between passbands, but they do not actually suggest determining separate transformation terms for each pixel.

An additional complication is that the active volume changes with the exposure level (Percival and Nordsieck 1980, Kesler and Lomheim 1986). Therefore the contrast and positioning of the fringes depends on exposure. This probably explains the difficulties often experienced in trying to subtract night-sky fringes; they are not a purely additive phenomenon, and so do not "subtract" accurately. Neither are they fixed-position sensitivity variations that can be divided out in flatfielding. One can only agree with Gullixson (1992) that, for accurate work, "just say no to CCDs that produce fringes in dome flatfields." I would go farther and just say no to CCDs that produce fringes in night-sky exposures.

The dependence of responsivity on exposure makes CCDs inberently non-linear, in a way that depends on the particular filter used and position on the chip. As the time-dependent active volume depends on voltages, clocking frequencies and waveforms, one expects the nonlinearity to depend on the electronic settings, as was actually found by Gosset and Magain (1993).

Indeed, on close examination, about $1 / 3$ of the CCDs investigated by Abbott and Sinclaire (1993) showed nonlinearities exceeding one percent. Similar results were obtained by Gilliland et al. (1993). In addition, the latter authors remark that "Most CCDs have at least isolated regions that respond nonlinearly to light at low intensity." This is analogous to the "inertia" of photographic plates, though the mechanism is different in the two detectors. Other wavelength-dependent nonlinear effects are known, even in simple photodiodes (Schaefer et al. 1983). Unfortunately, because the nonlinearity is wavelength-dependent, one cannot exactly define a spectral-responsivity function for a CCD. Such properties make CCDs look remarkably like those the detectors they have largely replaced: photographic plates.

\section{COMPARISONS TO PHOTOGRAPHY}

The careful investigations of CCD properties mentioned above have shown that CCDs share many of the properties of photographic plates, such as wavelength-dependent nonlinearity. The problems arising from internal standing waves are inherent in the optical physics of the devices, and are not due to "manufacturing defects" that can be eliminated; major changes in device design and technology would be required to avoid these problems. To be sure, the problems with CCDs are about an order of magnitude smaller than those of photography. Nevertheless, those who require accuracy in their data may come to regard CCD photometry much as photographic photometry is regarded today: a sort of second-rate substitute for the real thing.

Indeed, at the NATO Workshop on Long-Term Photometry of Variable Stars in Ghent last November, I was surprised to hear so many critical remarks about CCDs in the corridor discussions. I have heard similar remarks from laboratory photometrists, to the effect that no one should ever expect CCDs to produce as good results as do PMTs. There is the danger that 
CCDs were over-sold at first by their enthusiasts, and that a backlash is developing because of the continued difficulty in demonstrating really accurate results from CCDs.

If CCDs are to earn the full confidence of the photometric community, attention must be paid to their optical properties as well as their electrical ones. Not only antireflection coatings, but even changes in basic device design and construction may be required to eliminate problems due to internal fringes.

In any case, it appears that just as much care is required to extract good photometry from $\mathrm{CCDs}$ as is required in photographic photometry. Because many of the problems that arise are similar, it would be useful for CCD observers to study the problems long known to exist with photography, to avoid wasting effort on problems that have previously been well studied.

\section{OTHER OPTICAL PROBLEMS}

Some flatfielding problems clearly can be traced to stray light (Tobin 1993, Walker 1993). Proper baffling in telescopes, and light-tight camera construction, are required to avoid these problems. Tobin et al. (1993) have shown that filter nonuniformities are significant; filters near the focal plane must be repositioned to better than 100 microns to avoid measurable errors.

One should also remember that the periodic structure of a CCD forms a diffraction grating. There should therefore be considerable polarization effects, particularly at wavelengths where a diffracted order passes off the surface of the grating; because the grating is relatively coarse, several such anomalies are to be expected within a broad photometric passband. Furthermore, the interference effects within each pixel may differ depending on the polarization state. Tobin (1993) reports pixel-to-pixel polarization effects between 0.7 and $3 \%$, but more work on this problem is clearly needed.

\section{DISCUSSION}

The need to tailor filters to individual CCD chips requires substantial operational changes at most observatories. First of all, accurate laboratory calibration facilities are needed to measure the spectral responses of individual detectors; but such facilities are uncommon today, even at large observatories.

Second, the idea of "standard" filter sets must be abandoned; filters cannot be moved from one instrument to another with impunity. Designing filters for each chip requires measuring individual glass samples, and finishing them to the correct thickness, because catalog tolerances on Schott sharp-cutoff glasses correspond to a factor of more than two on either side of the nominal catalog thickness. Photometric filters should never be ordered by glass type and thickness alone.

In narrowband work, the passband can be a fraction of an Edser-Butler channel in width. Then one might improve accuracy by determining the local spectral slope for each pixel. Efforts should be made to develop calibration techniques to do this. However, it would still be necessary to design filters to take account of each CCD's average spectral response.

Even with optimal filters for each chip, spatial variations contribute substantial conformity 
errors. The obvious way to reduce the effect of pixel-to-pixel differences in spectral response is to average over a large number of pixels, as was done by Gilliland et al. (1993), who essentially resorted to Fabry photometry - a lesson learned from the problems of photographic photometry. In wideband photometry, these variations apparently set a fundamental limit to the accuracy that can be achieved with direct imaging. One should always bear in mind Fred Vrba's remark: "Imaging is not photometry."

This work was partly supported by NSF Grant AST-8913050.

\section{REFERENCES}

Abbott, T. M. C. and Sinclaire, P. 1993 The Messenger 73, 17

Beckert, D. C. and Newberry, M. V. 1989 PASP 101, 849

Bessell, M. S. 1990 PASP 102, 1181

Bessell, M. S. 1993a in Stellar Photometry - Current Techniques and Future Developments, C. J. Butler and I. Elliott, eds., Cambridge University Press, Cambridge, p. 22

Bessell, M. S. 1993b in Precision Photometry, D. Kilkenny, E. Lastovica, and J. W. Menzies, eds., South African Astronomical Observatory, Capetown, p. 46

Geist, J., Gladden, W. K., and Zalewski, E. F. 1982 J. Opt. Soc. Amer. 72, 1068

Gilliland, R. L., Brown, T. M., Duncan, D. K., Suntzeff, N. B., Lockwood, G. W., Thompson, D. T., Schild, R. E., Jeffrey, W. A. and Penprase, B. E. 1991 AJ 101, 541

Gilliland, R. L., Brown, T. M., Kjeldsen, H., McCarthy, J. K., Peri, M. L., Belmonte, J. A., Vidal, I., Cram, L. E., Palmer, J., Frandsen, S., Parthasarathy, M., Petro, L., Schneider, H., Stetson, P. B., and Weiss, W. 1993 AJ 106, 2441

Gosset, E., and Magain, P. 1993 The Messenger 73, 13

Gullixson, C. A. 1992 in Astronomical CCD Observing and Reduction Techniques, S. B. Howell, ed., ASP, San Francisco, p. 130

Gunn, J. E. and Stryker, L. L. 1983 ApJS 52, 121

Kesler, M. P., and Lomheim, T. S. 1986 Appl. Opt. 25, 3653

King, I. 1952 AJ 57, 253

Manfroid, J. and Sterken, C. 1992 A\&A 258, 600

Massey, P. and Jacoby, G. H. 1992 in Astronomical CCD Observing and Reduction Techniques,. S. B. Howell, ed., ASP, San Francisco, p. 240

Percival, J. W. and Nordsieck, K. H. 1980 PASP 92, 362

Schaefer, A. R. 1984 in Workshop on Improvements to Photometry, W. J. Borucki and A. T. Young, ed., NASA Ames Research Center, Moffett Field, p. 193

Schaefer, A. R., Zalewski, E. F. and Geist, J. 1983 Appl. Opt. 22, 1232

Schumann, L. W. and Lomheim, T. S. 1989 Appl. Opt. 28, 1701

Tobin, W., Kershaw, G. M., Ritchie, R. A., Ma, L., Graham, G. J. and Hemmingsen, S. B. 1993 in Poster Papers on Stellar Photometry, I. Elliott and C. J. Butler, eds., Dublin Institute for Advanced Studies, Dublin, p. 304

Tobin, W. 1993 in Stellar Photometry - Current Techniques and Future Developments, C. J. Butler and I. Elliott, eds., Cambridge University Press, Cambridge. p. 304

Walker, A. R. 1993 in Stellar Photometry - Current Techniques and Future Developments, C. J. Butler and I. Elliott, eds., Cambridge University Press, Cambridge, p. 278

Young, A. T. 1992a A\&A 257, 366

Young, A. T. $1992 \mathrm{~b}$ in Automated Telescopes for Photometry and Imaging,, S. J. Adelman, R. J. Dukes, and C. J. Adelman, eds., ASP Conf. Series, San Francisco, p. 73 
Young, A. T. 1994 A\&A, 288, 683

\section{DISCUSSION}

OSWALT: Where in relative importance does the lack of faint and lack of red standard stars fall compared to other sources of error in transforming CCD observations to a standard system? At present stars can be observed which are at least several magnitudes fainter and at least 1 2 mag redder [in (V-I)] than published lists of standard stars.

YOUNG: This is a big problem, especially for the $M$ stars that appear in great numbers at faint magnitudes. Arlo Landolt has been working on faint CCD standards.

JORDEN: You discussed "fringes" and showed curves of CCD spectral response for a frontside CCD. I consider that the term "fringing" normally refers to long wavelength internal fringes $(\lambda>700 \mathrm{~nm})$. In backside CCDs, the variation of spectral response for a thick, frontside CCD is somewhat different.

YOUNG: The fringes are the spatial alias of Edsen-Butler fringes (standing waves) inside the chip, and occurs for both front and back side illumination. They are usually worse for front side illumination.

JORDEN: Do you consider that when astronomers publish photometric data, they should also include the spectral response of the CCD and filter transmission characteristics?

YOUNG: Absolutely!

TOBIN: The CCD quantum efficiency is a crucial part of the passband. How accurate are the $\mathrm{QE}$ curves supplied by manufacturers, especially with respect to shape?

YOUNG: I can't say from my own experience; but my impression from published work is that manufacturers' curves are only a rough guide - perhaps accurate only to some tens of percent.

STERKEN: The shortcomings in photometry are not only the CCD and the filters! Besides some of the problems illustrated there is a huge additional shortcoming in education and training of students as photometrists - that is, as well from the point of view of understanding transformation, as from the point of view of observing experience. Enhanced by the all-present high tech data acquisition and reduction tools, larger errors are introduced of non-detector origin than by the whole CCD camera itself. The CCD is becoming a very reliable detector; it is the human interface that is a problem.

YOUNG: Yes, users need to be well trained in the basics of photography. Experience with photographic photometry is very useful, as many of the same problems occur with CCDs, but at a lower level. Photometry shows you what to watch out for.

TOBIN: I've found large differences in UV Schott glasses.

FLORENTIN-NIELSEN: Concerning the QE response, it is not so difficult to measure 
absolute QE (at say 10 wavelengths) of the CCD. It is particularly important in case you operate UV flooded CCDs when UV response may vary considerably with time. 